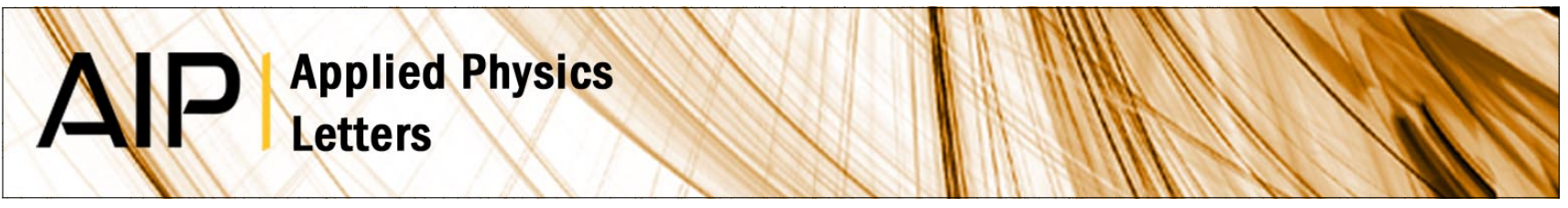

\title{
Distinct band gaps and isotropy combined in icosahedral band gap materials
}

D. Sutter-Widmer, P. Neves, P. Itten, R. Sainidou, and W. Steurer

Citation: Appl. Phys. Lett. 92, 073308 (2008); doi: 10.1063/1.2884319

View online: http://dx.doi.org/10.1063/1.2884319

View Table of Contents: http://apl.aip.org/resource/1/APPLAB/v92/i7

Published by the American Institute of Physics.

\section{Related Articles}

Needle-like focus generation by radially polarized halo beams emitted by photonic-crystal ring-cavity laser Appl. Phys. Lett. 101, 221103 (2012)

Observation of quantum Talbot effect from a domain-engineered nonlinear photonic crystal Appl. Phys. Lett. 101, 211115 (2012)

Čerenkov nonlinear diffraction in random nonlinear photonic crystal of strontium tetraborate Appl. Phys. Lett. 101, 211114 (2012)

Self-induced spin-polarized carrier source in active photonic device with artificial optical chirality Appl. Phys. Lett. 101, 181106 (2012)

Nanocrystalline diamond photonics platform with high quality factor photonic crystal cavities Appl. Phys. Lett. 101, 171115 (2012)

\section{Additional information on Appl. Phys. Lett.}

Journal Homepage: http://apl.aip.org/

Journal Information: http://apl.aip.org/about/about_the_journal

Top downloads: http://apl.aip.org/features/most_downloaded

Information for Authors: http://apl.aip.org/authors

\section{ADVERTISEMENT}

\section{AIP Applied Physics Letters}

\section{EXPLORE WHAT'S NEW IN APL}

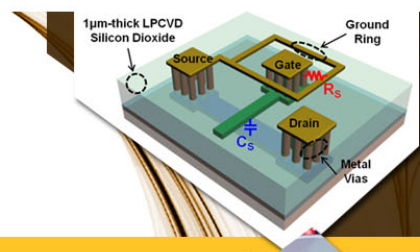

SURFACES AND INTERFACES

Focusing on physical, chemical, biological, structural, optical, magnetic and electrical properties of surfaces and interfaces, and more.. 


\title{
Distinct band gaps and isotropy combined in icosahedral band gap materials
}

\author{
D. Sutter-Widmer, ${ }^{1, a)}$ P. Neves, ${ }^{1}$ P. Itten, ${ }^{1}$ R. Sainidou, ${ }^{2}$ and W. Steurer ${ }^{1}$ \\ ${ }^{1}$ Laboratory of Crystallography, Department of Materials, ETH Zurich, 8093 Zurich, Switzerland \\ ${ }^{2}$ Instituto de Óptica-CSIC, Serrano 121, 28006 Madrid, Spain
}

(Received 7 December 2007; accepted 31 January 2008; published online 21 February 2008)

\begin{abstract}
Icosahedral band gap materials (BGMs) optimally combine the distinct band gaps of periodic BGMs with the high rotational symmetry of quasiperiodic structures. This is shown experimentally for longitudinal and transverse polarized elastic waves in a phononic crystal based on the three-dimensional Penrose tiling (3D-PT) and applies equally to photonic crystals. The ability of icosahedral BGMs to form Bragg-type band gaps follows from the similarity between the 3D-PT and the face-centered cubic structure (its periodic average structure). The 3D quasiperiodic BGM lacks bands of strong transmission like random or disordered BGMs but shows clear band gaps like periodic BGMs do. (C) 2008 American Institute of Physics. [DOI: 10.1063/1.2884319]
\end{abstract}

Phononic (photonic) crystals (PNCs) are composite materials which strongly interact with elastic (electromagnetic) waves. They are artificially structured with typical distances much larger than the atomic scale. The sizes of the unit building blocks (scatterers) constituting these mesostructures (i.e., spheres, rods, or sheets embedded in a different matrix material) are chosen to match any wavelength of interest. Bragg scattering and resonances of the single scatterers both allow the formation of bands and gaps just as for electrons in crystals. Well-defined band gaps predestine such composites to be used as wave shields or filters. Defect modes arising from disturbing the ordered structures open the way for applications such as waveguides or cavities. Band gap materials (BGMs) have therefore been intensively studied in the last years. ${ }^{1}$

The inherent structural anisotropy of periodic BGMs, which show at best cubic symmetry, hinders the formation of omnidirectional band gaps. This has been the motivation for the study of the much more complex but higher-symmetric quasiperiodic BGMs (for review, see Ref. 2). Quasiperiodic structures show pure-point Fourier spectra as periodic structures do. The set of all scattering vectors of a periodic structure forms the reciprocal lattice. That of a quasiperiodic structure forms a Z module $M^{*}$ of rank $N$, with $N$ larger than the dimension $d$ of the structure. In this case, the Bragg reflections form a dense set. The closer to each other these Bragg reflections are, the more they differ in intensity. This means that Bragg peaks with intensities above a given threshold value always form discrete diffraction patterns. Each set of symmetrically equivalent, strong Bragg peaks forms a pseudo-Brillouin zone of arbitrarily high symmetry, which can induce a band gap.

While gap formation due to resonances of single scatterers is straightforwardly analogous to that in periodic BGMs, ${ }^{3,4}$ the formation of Bragg gaps in quasiperiodic heterostructures has been much discussed. ${ }^{5,6}$ We have previously shown that the periodic average structure (PAS) is helpful for predicting the potential of two- dimensional (2D) quasiperiodic structures for Bragg gap formation in BGMs. The PAS gives a measure of the average degree of periodicity present in a quasiperiodic structure and also characterizes the type of deviation (displacive or substitutional). A PAS is defined via its Fourier transform, a reciprocal lattice spanned by three strong Bragg peaks of the Fourier transform of the quasiperiodic structure. ${ }^{7}$ While the other common concept to simplify quasiperiodic structures, the approximants (periodic structures containing local motifs of a quasiperiodic structure), leads to structures with still several hundred sites per unit cell, the PAS is simply a Bravais lattice. We apply here this procedure, which treats the quasiperiodic BGM as a disordered periodic one, on a three-dimensional (3D) icosahedral BGM.

The few studies on icosahedral BGMs published so far mostly focus on production techniques of photonic quasicrystals with network topologies. ${ }^{8-13}$ They provide theoretical evidence for the formation of gaps due to resonances $^{9}$ in three-dimensional Penrose tiling (3D-PT) based BGMs and experimental evidence for Bragg diffraction based gaps. ${ }^{10,14}$ The latter were also used to redraw the effective zone boundary scheme of the 3D-PT, which appeared to be astonishingly simple and closely related to that of a face-centered cubic (fcc) BGM. ${ }^{10}$ That this similarity is just a consequence of the relationship between a quasiperiodic structure and its PAS will be shown in the following on a 3D-PT based quasiperiodic phononic crystal (QPNC). The icosahedral 3D-PT is a nonperiodic arrangement of two types of prototiles (a prolate and an oblate rhombohedron with edge lengths $a_{r}$ ), and it is the only 3D quasiperiodic structure that has been studied in detail. ${ }^{15}$ The vertices of a section of the tiling are shown in Fig. 1(b). The point set can be generated as an intersection of a six-dimensional (6D) primitive hypercubic structure, decorated by triacontahedral atomic surfaces (occupation domains), with 3D physical space (a 3D subspace invariant under icosahedral point group operations). The $6 \mathrm{D}$ basis of the tiling ${ }^{7}$ is defined by

\footnotetext{
${ }^{a)}$ Electronic mail: daniel.sutter@mat.ethz.ch.
} 

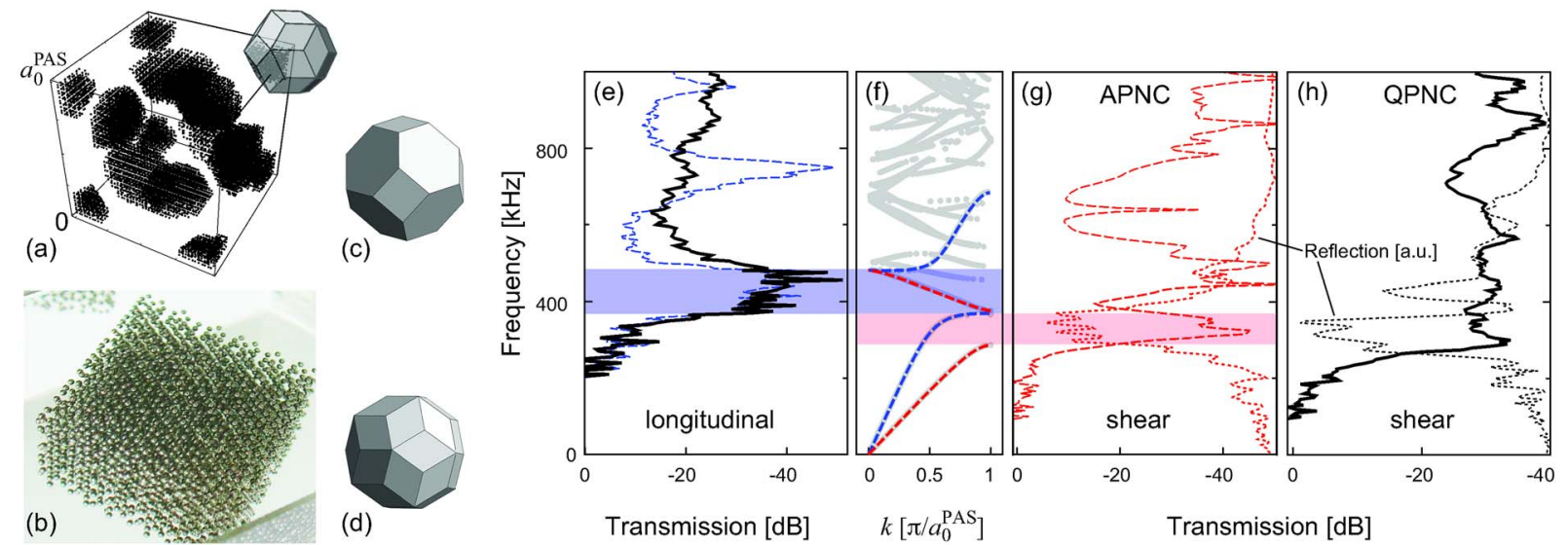

FIG. 1. (Color online) The icosahedral tiling modulo the unit cell of the PAS results in point sets circumscribed by triacontahedra on a fcc lattice (a). The QPNC consisting of steel beads in polyester (b). Shapes of the (pseudo) zone boundaries of the fcc (c) and the icosahedral (d) structures. Experimental longitudinal wave transmission spectra of the QPNC along a twofold axis (solid line) and its APNC for the [100] direction (dashed line) (e). Calculated band structure of the APNC along the [100] direction (f). Transmission (dashed line) and reflection (dotted line) spectra for shear waves for the APNC (g) and QPNC (h) [directions as in (e)]. The shaded regions in (e)-(g) denote the frequency ranges of the longitudinal hybridization and the shear Bragg gaps.

$$
\begin{gathered}
\mathbf{d}_{1}=a_{r}\left(\begin{array}{c}
0 \\
0 \\
1 \\
0 \\
0 \\
1
\end{array}\right), \quad \mathbf{d}_{i}=a_{r}\left(\begin{array}{c}
s \cos (2 \pi i / 5) \\
s \sin (2 \pi i / 5) \\
c \\
-s \cos (4 \pi i / 5) \\
-s \sin (4 \pi i / 5) \\
-c
\end{array}\right) \\
\text { for } i=2, \ldots, 6,
\end{gathered}
$$

with $s=\sin \theta, c=\cos \theta$, and $\theta=63.44^{\circ}$. The atomic surfaces have no extension in physical space but generate the tiling vertices by intersecting with it. The oblique projection of the 6D lattice and the triacontahedra along the three directions

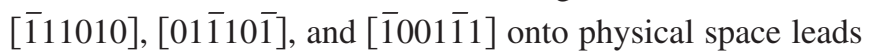
to a fcc PAS with the basis set $\mathbf{a}_{1}=a_{r}(\tau-1,0, \tau-2), \mathbf{a}_{2}$ $=a_{r}[0, \tan (\pi / 5), 0], \quad$ and $\mathbf{a}_{3}=a_{r}(\tau-2,0,1-\tau)$, where $\tau$ $=1.618$ is the golden mean. The lattice parameter ${ }^{7}$ is $a_{0}^{\mathrm{PAS}}$ $=2 \tan (\pi / 5) a_{r}$. The three projectors are determined by the three strong Bragg peaks in the Fourier transform of the tiling, which define the PAS. The projections in real space correspond to cutting the reciprocal PAS lattice from the $\mathbb{Z}$ module $M^{*}$. The oblique projections of the atomic surfaces are undistorted triacontahedra at vertices of a fcc lattice filling $\alpha_{a}=19.5 \%$ of the volume of its unit cell [see Fig. 1(a)]. The deviation of the tiling from its PAS is best illustrated by superposing the two structures. All tiling vertices then fall inside a projected atomic surface, while $18 \%$ of these contain two vertices (i.e., the occupancy of the projected atomic surfaces is $\left.\rho_{\text {occ }}=1.18\right)$. The maximal displacement of a tiling vertex from the PAS, $\alpha_{\max }=0.26 a_{0}^{\mathrm{PAS}}$, equals the circumradius of the triacontahedra.

We have manufactured two PNCs of steel spheres (1 mm diameter) embedded in polyester, one with the structure of a 3D-PT (QPNC, a PNC made of 3438 spheres) and the other with that of its fcc PAS [(APNC), a fcc PNC made of 1458 spheres]. For practical reasons, the minimal distance allowed between the centers of two adjacent spheres was chosen to be $1.25 \mathrm{~mm}$ [filling fractions, 0.062 (APNC) and 0.074 (QPNC); $a_{0}^{\mathrm{PAS}}, 3.227 \mathrm{~mm}$ ]. The construction evolved layerwise. The positions of a layer of spheres were fixed by holes drilled into a solid block of polyester. The holes were filled with spheres and overcast with liquid polyester, and after curing, the next layer of holes was drilled [see Fig. $1(\mathrm{~b})]$.

The calculated ${ }^{16}$ band structure of the APNC [Fig. 1(f)] along the [100] direction shows a hybridization band gap for longitudinal waves and a Bragg gap for transverse polarized waves. The resulting opaque frequency ranges are clearly visible in the transmission spectra. For longitudinal waves, the transmission curves of the QPNC and the APNC (measured in water with Panametrics V302 transducers) both indicate a deep and large band gap [see Fig. 1(e)]. This gap is a hybridization-induced gap: it opens up as a result of interaction between a narrow band originating from resonant dipole states of the individual spheres with a band describing the propagation of elastic waves in a homogeneous effective medium. ${ }^{17}$ Since both BGMs are constituted of almost equally-close-packed spheres of the same size, the resulting gaps of the QPNC and APNC coincide perfectly. The formation of this hybridization gap is quite insensitive to the structure $^{4}$ and the direction of transmission.

Quite the opposite is true for the first shear wave band gaps (measured with Panametrics V151 shear transducers), shown in Figs. 1(g) and 1(h). This gap is clearly of Bragg scattering origin, and the direction dependence of the gap region follows the shape of the 3D zone boundary [Fig. $1(\mathrm{c})]$. The overlap of the gaps for the [100] and [111] directions of the APNC is only about $50 \%$. The Bragg gaps of the QPNC are much more isotropic due to the higher symmetry of its pseudo-zone-boundary [Fig. 1(d)]. The higher symmetry is also illustrated by the order of the point groups, which is 120 in case of the icosahedral group $m \overline{3} \overline{5}$, compared to 48 for the cubic group $m \overline{3} m$. While the bands and gaps of the APNC are clearly visible in both transmission and reflection spectra [Fig. 1(g)], the Bragg gap formation of the QPNC is indicated only in the reflection spectrum [Fig. 1(h)]. A reflection band can be seen, which is distinct for a quasiperiodic BGM and coincides well with that of the APNC. The central frequencies are equal because the zone boundaries inducing the gap are common to the two structures. It is interesting to note that for the QPNC, the frequency ranges of low reflection amplitudes do not directly correlate with ranges of high transmission amplitudes. Apart from the low frequency band, 
the QPNC does not allow transmission on a level as high as that of the APNC. The existence of a band gap, however, is clearly visible in the reflection spectrum.

In the following, we discuss how the agreement of these first shear wave Bragg gaps of the two PNCs relates to their structural similarity. The deviation of the 3D-PT from its PAS is described above by the parameters $\alpha_{a}=0.195$ and $\rho_{\text {occ }}=1.18>1$. In the absence of other prominent 3D tilings, we compare $\alpha_{a}$ to those of 2D tilings. The 2D octagonal tiling $\left(\alpha_{a}=0.19, \rho_{\text {occ }}=0.82\right)$ is well described by its square PAS, whereas the 2D-PT $\left(\alpha_{a}=0.34, \rho_{\text {occ }}=0.85\right)$ deviates significantly from its rhombic PAS. ${ }^{6}$ While octagonal QPNCs revealed clear bands and gaps, 2D Penrose QPNCs were found to transmit waves more like a disordered periodic PNC. ${ }^{6}$ If $\alpha_{a}$ is corrected for the dimensionality $d$ as $\alpha_{a}^{1 / d}$, then the 3D-PT reaches almost the same value (and therewith the same degree of displacive deviation from the PAS), 0.580, as the 2D-PT (0.583), which is higher than that of the octagonal tiling (0.436). Now, deviations from ordered structures tend to localize waves. The tendency is thereby stronger if deviation from order is large, but it reduces with increasing dimensionality as well as with decreasing scattering strength. In $3 \mathrm{D}$ the defect potential has to exceed a certain minimal threshold for localization to occur at all. In $1 \mathrm{D}$ and $2 \mathrm{D}$, on the other hand, any defect localizes some wave states. ${ }^{18}$ If we treat here the QPNC as a disordered fcc PNC, then the influence of this disorder can be expected to affect wave propagation less than it does in 2D. Localized states lead to many weak peaks in the transmission spectrum and vanishing of clear bands and gaps. The maximal transmission of bands of the APNC, on the other hand, decreases smoothly toward higher frequencies, illustrating the influence of absorption. Thus, the icosahedral QPNC can be considered as quite aperiodic (as its 2D analog), which causes suppression of strongly transmitting bands. However, since the aperiodicity is less effective in 3D in terms of localization, the larger correlation length allows the formation of clear band gaps, as in its APNC.

Our investigation demonstrates the usefulness of PAS for the prediction of Bragg gaps in 3D QPNCs and explains the similarity between the reflection properties of icosahedral and fcc PNCs. Now that manufacturing processes are estab- lished, 3D quasiperiodic BGMs can become very important because they are highly isotropic and still retain the clear gaps of periodic BGMs, i.e., more than 1D and 2D QPNCs, 3D QPNCs act similar to periodic PNCs but with just higher symmetry. BGMs with quasiperiodic structures (structures often described as intermediate between random and periodic ones) transmit waves like random heterostructures do, but they do form band gaps (of both hybridization and Bragg origin), just as periodic BGMs do. With regard to applications, this makes such materials unfavorable as wave filters but very interesting for mirroring, trapping, and guiding of waves.

We would like to thank Hans Reifler and Werner Blattmann for their help with sample preparation.

\footnotetext{
${ }^{1}$ M. Sigalas, M. S. Kushwaha, E. N. Economou, M. Kafesaki, I. E. Psarobas, and W. Steurer, Z. Kristallogr. 220, 765 (2005).

${ }^{2}$ W. Steurer and D. Sutter-Widmer, J. Phys. D 40, R229 (2007).

${ }^{3}$ C. Rockstuhl, U. Peschel, and F. Lederer, Opt. Lett. 31, 1741 (2006).

${ }^{4}$ D. Sutter-Widmer and W. Steurer, Phys. Rev. B 75, 134303 (2007).

${ }^{5}$ M. A. Kaliteevski, S. Brand, R. A. Abram, T. F. Krauss, R. DeLa Rue, and P. Millar, Nanotechnology 11, 274 (2000).

${ }^{6}$ D. Sutter-Widmer, S. Deloudi, and W. Steurer, Phys. Rev. B 75, 094304 (2007).

${ }^{7}$ W. Steurer and T. Haibach, Acta Crystallogr., Sect. A: Found. Crystallogr. 55, 48 (1999).

${ }^{8}$ M. Duneau and M. Audier, Acta Crystallogr., Sect. A: Found. Crystallogr. 55, 746 (1999).

${ }^{9}$ S. K. Cheung, T. L. Chan, Z. Q. Zhang, and C. T. Chan, Phys. Rev. B 70, 125104 (2004).

${ }^{10}$ W. Man, M. Megens, P. J. Steinhardt, and P. M. Chaikin, Nature (London) 436, 993 (2005).

${ }^{11}$ Y. Roichman and D. G. Grier, Opt. Express 13, 5434 (2005).

${ }^{12}$ A. Ledermann, L. Cademartiri, M. Hermatschweiler, C. Toninelli, G. A. Ozin, D. S. Wiersma, M. Wegener, and G. von Freymann, Nat. Mater. 5, 942 (2006).

${ }^{13}$ W. Y. Tam, Appl. Phys. Lett. 89, 251111 (2006).

${ }^{14}$ J. Xu, R. Ma, X. Wang, and W. Y. Tam, Opt. Express 15, 4287 (2006).

${ }^{15}$ J. E. S. Socolar and P. J. Steinhardt, Phys. Rev. B 34, 617 (1986).

${ }^{16}$ R. Sainidou, N. Stefanou, I. E. Psarobas, and A. Modinos, Comput. Phys. Commun. 166, 197 (2005).

${ }^{17}$ R. Sainidou, N. Stefanou, and A. Modinos, Phys. Rev. B 66, 212301 (2002).

${ }^{18}$ E. N. Economou, Green's Functions in Quantum Physics (Springer, Ber-
} lin, 1979). 